\title{
Semen parameter improvements after microsurgical subinguinal varicocele repair are durable for more than 12 months
}

Vinayak Madhusoodanan; Premal Patel, MD; Ruben Blachman-Braun, MD; Ranjith Ramasamy, MD

University of Miami Miller School of Medicine, Miami, FL, United States

Cite as: Can Urol Assoc J 2019 September 27; Epub ahead of print. http://dx.doi.org/10.5489/cuaj.6047

Published online September 27, 2019

$* * *$

\section{Abstract}

Introduction: Varicoceles account for the most common correctable cause of male infertility, with varicocele repair leading to improvements in semen quality. However, there is little evidence to establish the durability of varicocele repair. We analyzed the durability of improvements in postoperative semen parameters following microsurgical subinguinal varicocele repair.

Methods: We evaluated all men who underwent microscopic subinguinal varicocelectomy from 2015-2019. Patients were included if they desired fertility and had a followup of at least 12 months. We assessed the baseline characteristics of these patients, as well as semen volume, total motile sperm count (TMSC), concentration, percent motility, and morphology. Semen parameters were analyzed at baseline (preoperative), approximately three months and $\geq 12$ months postoperatively.

Results: Of 105 men who underwent varicocelectomy, 18 men had a followup of at least 12 months. These men presented with median age 34.5 (27-38] years for a median followup duration of 14.5 (13-22.5) months. TMSC levels increased from 6.4 (1.1-24.5) million at baseline to $11.1(2.4-38.4)$ million at approximately three months and remained similar at 12.5 (1.6-31.5) million at $\geq 12$ months. The study is limited by its retrospective nature and limited sample size.

Conclusions: Microscopic subinguinal varicocele repairs can result in durable improvements of semen quality beyond one year, as demonstrated by upgrade in median TMSC. Further studies should be performed to confirm our findings. 


\section{Introduction}

A varicocele is a dilation of the pampiniform venous plexus, this pathology has a prevalence equivalent to approximately $15 \%$ of males, and it can be clinically relevant in up to $20 \%$ of that population.$^{1-3}$ Its effect on semen parameters was first described in 1965 by Macleod, and although largely asymptomatic, it stands the most common correctable cause of male infertility, affecting up to $41 \%$ of men with primary infertility, up to $81 \%$ of men with secondary infertility and up to $45 \%$ of men with dyspermia. ${ }^{1-3}$

It is largely agreed upon that varicoceles can result in testicular hypotrophy, gonadotropin level changes and impaired spermatogenesis. ${ }^{2,3}$ Most investigations of varicocele pathophysiology propose a mechanism of impaired testicular blood flow, which can result in increased scrotal temperature. Although the specific pathophysiology leading to impaired spermatogenesis remains elusive, numerous studies have shown varicocele repair to be effective in improving pregnancy rate through improvements in semen quality, especially in regards to semen motility and concentration. ${ }^{2}$

For adults presenting with infertility and varicocele, the benefit of varicocelectomy is clear. ${ }^{4}$ Repairing clinical varicoceles in oligospermic and non-obstructed azoospermic men prior to in-vitro fertilization (IVF) can be beneficial, and has been shown to decrease levels of assisted reproductive technology (ART) necessary to achieve successful pregnancy in both subclinical and clinical varicoceles. ${ }^{5-7}$ However, for adolescents, the decision to treat is controversial as the goal of management becomes preventing testicular injury and maintaining function for future fertility. ${ }^{4}$ Techniques for repair include retroperitoneal, laparoscopic, inguinal and subinguinal approaches, but subinguinal, specifically when aided by an operating microscope, are favored in adults while laparoscopic approaches are favored in adolescents. ${ }^{2,4,8}$ In both adults and adolescents, identifying who will likely benefit, and for how long from repair, remains a topic of further investigation. ${ }^{4}$

Despite the wealth of evidence demonstrating the clinical benefit of varicocele repair, most studies follow patients at 3-month intervals for a maximum of 6-12 months. ${ }^{8,9}$ Scant literature exists to qualify durability of such improvement at periods $\geq 12$ months. The aim of this study is to analyze the durability of improvements in post-operative semen parameters following microsurgical varicocele repair. We hypothesized subinguinal varicocelectomy will yield durable results at 1-year post-operative.

\section{Methods}

An IRB approval was acquired, and a retrospective chart review was performed, including all patients who underwent microsurgical subinguinal varicocelectomy between August 2015 and October 2018. All procedures were performed by a single surgeon, and varicoceles for repair were clinically palpable or subclinical (i.e. detected by ultrasound).

All patients underwent a thorough evaluation that consisted of a physical exam, hormonal profile (follicular stimulating hormone, luteinizing hormone, and testosterone) and semen 
analysis. Physical exam was used to determine testicular volume, orchidometer-based measurement, in cubic centimeters (cc), laterality and varicocele grade in accordance with the physical exam. Patients underwent two preoperative semen analyses, the mean of which was used to establish baseline preoperative semen parameters. The following semen analysis parameters were recorded: volume, total motile sperm count (TMSC), concentration, percent motility, and morphology. On follow-up after surgery, semen analyses were obtained at $\sim 3$ month intervals. Prior to providing semen analysis samples, patients were instructed to remain abstinent for a minimum of 2 days. A single lab technician performed both semen analyses for all patients to minimize inter-observer variability.

Patients included in the study presented with chronic orchialgia or desired fertility with varicocele, and those with a recorded follow-up with semen analyses $<12$ months were excluded. These patients were subsequently studied to observe changes in their semen parameters over time-at baseline (pre-operative), 3 months, 6 months, 9 months and any follow-up $\geq 12$ months post-operatively, however, due to inconsistent post-operative follow-up, we have only presented here results from baseline, follow-up at $\sim 3$ months and follow-up $\geq 12$ months. TMSC at each period was of interest due to its utility in grading patient eligibility for ART, and morphology was omitted from results due to the inconsistent collection at follow-up.

For the statistical analysis, continues variables were presented as means and standard deviations ( \pm SD) or medians and interquartile ranges [IQR 25-75] according to the data distribution. Comparison of semen parameters values was performed using the Mann-Whitney U or KruskalWallis test as required. Categorical variables were presented as absolute values and frequencies. For this research a p-value $<0.05$ was considered statistically significant.

\section{Results}

The study analyzed 18 men who underwent microscopic subinguinal varicocelectomy. For these men, the median age at surgery was 34.5 [27 - 38], mean testicular volume was 14 [12.5-14.8] cc, bilateral varicocele was present in $5(27.8 \%)$ of patients, and the distribution of varicoceles by grade were subclinical $=2(11.1 \%), \mathrm{I}=2$ (11.1\%), II $=7$ (38.9\%) and III $=7$ (38.9\%). The median follow-up duration of the cohort was 14.5 [13 - 22.5] months (Table 1). When comparing semen parameters at these follow-up intervals, we note an improvement in TMSC, concentration and total motility from baseline to $\sim 3$ months, but the same was not observed of semen volume. TMSC and concentration were greater at $\sim 3$ and $\geq 12$ months postoperatively than at baseline. TMSC levels increased from 6.4 [1.1 - 24.5] million at baseline to 11.1 [2.4 - 38.4] million at $\sim 3$ months and 12.5 [1.6 - 31.5] million at $\geq 12$ months (Figure 1-2). Median and IQR of TMSC at both post-operative follow-up periods remain higher than that at baseline (Figure 2). Concentration increased from 10.7 [3.5 - 21.3] million sperm/cc semen at baseline to 14.5 [ 4 - 22.6] million sperm/cc semen at $~ 3$ months and 16 [1.4 - 20] million sperm/cc semen at $\geq 12$-months. 
However, it should be noted that these improvements in TMSC and semen concentration were not statistically significant. The comparative analysis did not find statistically significant differences for either $\sim 3$ month or $\geq 12$ month follow-up in comparison to baseline, with $\mathrm{p}=$ 0.650 for baseline vs. TMSC $\geq 12$ months (Table 2 ).

\section{Discussion}

In our study, we retrospectively evaluated 18 men desiring fertility who underwent subinguinal microsurgical varicocele repair and were followed up at 3-month intervals for at least 12 months. These patients were presented in terms of their baseline characteristics, baseline and postoperative semen parameters and duration of follow-up. We found that varicocele repair resulted in an improvement of semen quality, with improvement of both motility and concentration, that was maintained for a median duration of 14.5 months post-operative. However, it should be noted that due to the small sample size these changes were not statistically significant.

The clinical benefit of varicocele repair for clinical and subclinical varicoceles lies in its ability to improve spermatogenesis, and thereby reduce the need for ART. ${ }^{5-7}$ These studies have primarily used motility and concentration as markers for improvement in semen quality. ${ }^{2}$ Our study emphasizes these findings. In this study, TMSC improved from 6.4 [1.1 - 24.5] million at baseline to 11.1 [2.4 - 38.4] million at 3 months, and concentration improved from 10.7 [3.5 21.3] million sperm/cc semen at baseline to 14.5 [4 - 22.6] million sperm/cc semen at 3 months. These improvements were maintained at follow-up periods $\geq 12$ months, with TMSC of 12.5 [1.6 - 31.5] million and concentration of 16 [1.4 - 20] million sperm/cc semen.

These results showcase that varicocele repair can effectively improve TMSC from a median level at baseline indicating IUI (5-9 million sperm), to a median level at 3 and 12 months post-operative indicating natural pregnancy (>9 million sperm). ${ }^{5}$ It similarly demonstrated improvement in median semen concentration-10.7 million sperm/cc semen-that was well under normal ( $\geq 15$ million sperm/cc semen) at baseline to normal-16 million sperm/cc semen-at long-term follow-up ( $\geq 12$ months). The p-value of these changes in TMSC ( $p=0.65)$ and concentration ( $\mathrm{p}=0.56$ ) from baseline to long-term follow-up did not indicate statistical significance, but this should not be confused with clinical relevance, which has been established through "upgrade" in median TMSC and normalization of median concentration. ${ }^{5}$

Literature on the durability of post-varicocelectomy improvements in semen parameters is scarce. Existing studies focus on improvement of semen quality and pregnancy rate within 312 months of the repair. Masterson et al. described that men with TMSC $<5$ million can expect the largest improvement in TMSC within 3-6 months post-operatively, but only minimal improvement thereafter. ${ }^{8}$ Fukuda et al. found that after improvement from baseline to 3 months post-operative, there was no significant difference between semen parameters at 3 and 12 months post-varicocelectomy. ${ }^{10}$ The importance of our study lies in suggesting that these improvements in spermatogenesis are not transient. Although they may not increase substantially from levels at 3 months, they are certainly maintained well past this period, and past 1 year post-operative. 
Although our study has some strengths which include that physical exams and surgical procedures were performed by a single surgeon, and that the semen analysis was done by a single lab technician, some limitations include the inherent boundaries of a retrospective study, and a small sample size, in which several patients were lost to follow-up at either 3 or 6 months. This decreases the power of the study and affects its ability to reach statistically significant results.

Moreover, this study may have a selection bias, as patients with longer follow-up may have been persistently concerned with their fertility and patients who failed to follow-up with semen analysis for a period $\geq 12$ months might be those with the best response to the varicocelectomy, or may have achieved pregnancy. It is worth mentioning, that from the patients that were excluded $(n=39)$ due to incomplete follow-up, the median TMSC at 3 months postoperatively was 13.4 [5.0 - 18.2] million sperm, which was not statistically significant compared to the analyzed cohort ( $\mathrm{p}=0.948$ ). Although the TMSC reported during the same period was similar to that the 18 patients presented in our study, the semen parameter values and fertility rate are difficult to assess, and it might be possible that the excluded patients had a significant semen improvement after 3 months that was not measured. We expect other studies that include a wider range of semen parameters (i.e., semen reactive oxygen species and DNA fragmentation) to help better assess this. ${ }^{11}$

Furthermore, an improvement in semen quality may not translate into improved pregnancy rate. Thus, further multicentric studies that utilized a prospective methodology should be performed to assess microsurgical subinguinal varicocelectomy long-term changes in semen parameter (i.e., volume, TMSC, concentration, and total motility), semen reactive oxygen species and DNA fragmentation, pregnancy rate (both natural and assisted), and the effect on the hypothalamic-pituitary-gonadal axis. ${ }^{11}$

\section{Conclusions}

Our study suggests that microscopic subinguinal varicocele repair can result in clinically relevant improvements of semen quality that are durable in quality, and maintained for periods $\geq 12$ months post-operatively. Further studies should be performed to confirm our findings. 


\section{References}

1. Kohn TP, Kohn JR, Pastuszak AW. Varicocelectomy before assisted reproductive technology: are outcomes improved? Fertility and sterility 2017;108:385-91.

2. Zavattaro M, Ceruti C, Motta G, et al. Treating varicocele in 2018: current knowledge and treatment options. Journal of endocrinological investigation 2018;41:1365-75.

3. Clavijo RI, Carrasquillo R, Ramasamy R. Varicoceles: prevalence and pathogenesis in adult men. Fertility and sterility 2017;108:364-9.

4. Chiba K, Ramasamy R, Lamb DJ, Lipshultz LI. The varicocele: diagnostic dilemmas, therapeutic challenges and future perspectives. Asian journal of andrology 2016;18:27681.

5. Samplaski MK, Lo KC, Grober ED, Zini A, Jarvi KA. Varicocelectomy to "upgrade" semen quality to allow couples to use less invasive forms of assisted reproductive technology. Fertility and sterility 2017;108:609-12.

6. Thirumavalavan N, Scovell JM, Balasubramanian A, et al. The Impact of Microsurgical Repair of Subclinical and Clinical Varicoceles on Total Motile Sperm Count: Is There a Difference? Urology 2018;120:109-13.

7. Sedaghatpour D, Berookhim BM. The Role of Varicocele in Male Factor Subfertility. Current urology reports 2017;18:73.

8. Masterson TA, Greer AB, Ramasamy R. Time to improvement in semen parameters after microsurgical varicocelectomy in men with severe oligospermia. Canadian Urological Association journal = Journal de l'Association des urologues du Canada 2019;13:E66-e9.

9. Yazdani M, Hadi M, Abbasi H, et al. Efficacy of Varicocele Repair in Different Age Groups. Urology 2015;86:273-5.

10. Fukuda T, Miyake H, Enatsu N, Matsushita K, Fujisawa M. Assessment of Timedependent Changes in Semen Parameters in Infertile Men After Microsurgical Varicocelectomy. Urology 2015;86:48-51.

11. Roque M, Esteves SC. Effect of varicocele repair on sperm DNA fragmentation: a review. International urology and nephrology 2018;50:583-603. 


\section{Figures and Tables}

Fig. 1. Median total motile sperm count (in millions), with error bars representing interquartile range, observed at baseline, postoperative three months and postoperative $\geq 12$ months.

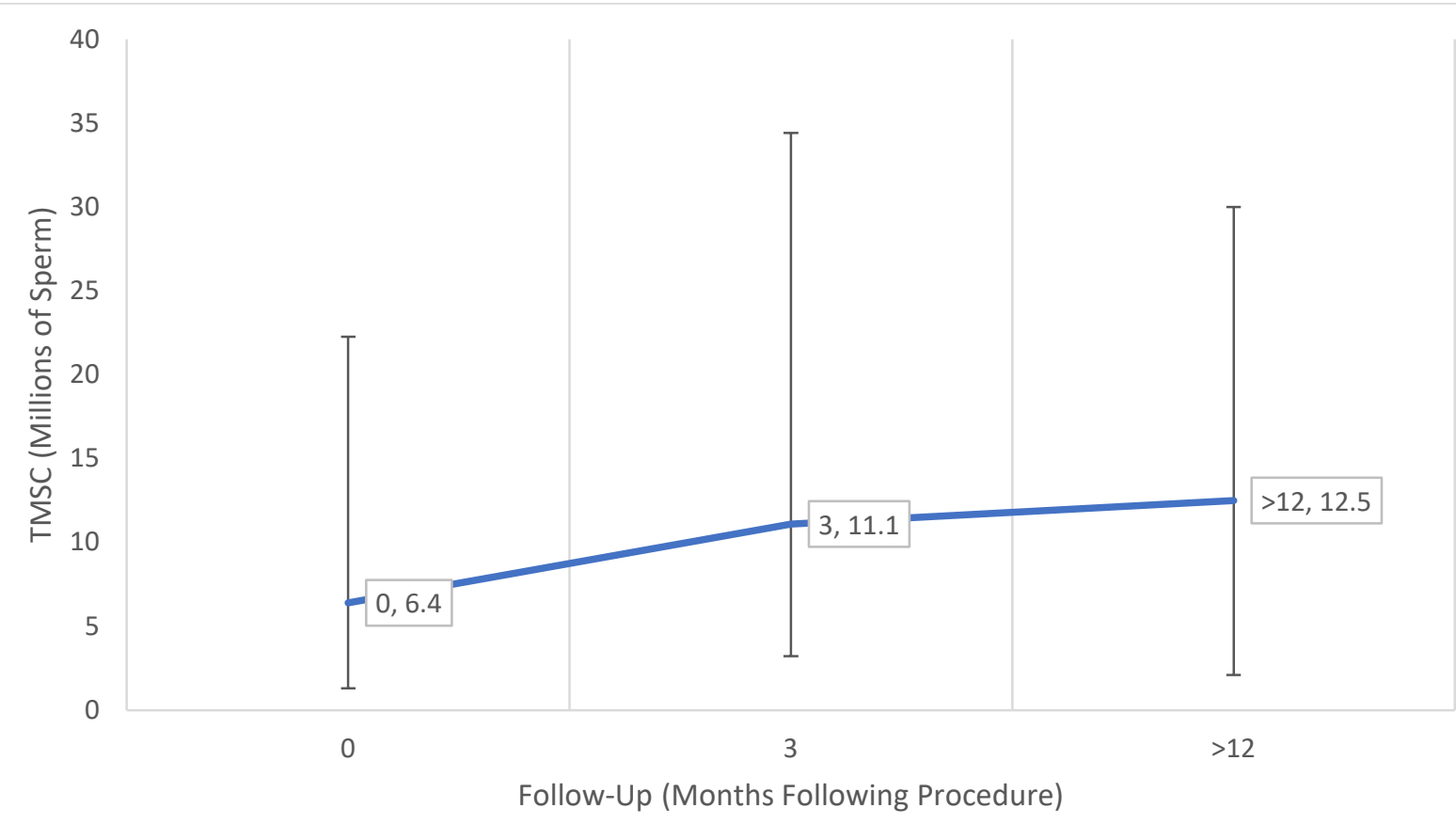


Fig. 2. Median and interquartile range of total motile sperm count (in millions) at baseline (preoperative) vs. postoperative $\geq 12$ months.

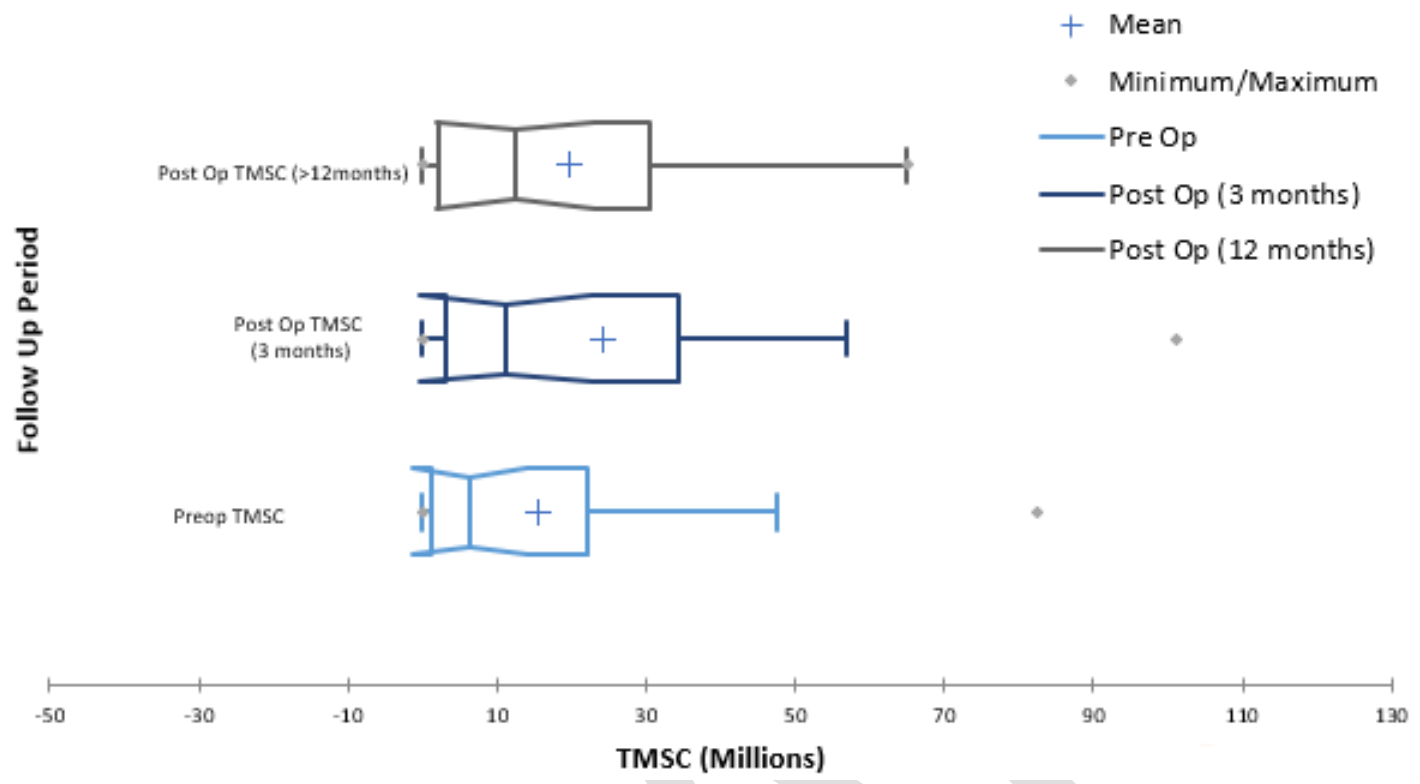

\begin{tabular}{|l|c|}
\hline \multicolumn{2}{|l|}{ Table 1. Demographic and clinical characteristics } \\
\hline & $\begin{array}{c}\text { Overall } \\
\mathrm{n}=18\end{array}$ \\
\hline Age at surgery in years & $33.6 \pm 8.9$ \\
\hline Laterality & $13(72.2)$ \\
\hline Left (\%) & $5(27.8)$ \\
\hline Bilateral (\%) & \\
\hline Higest grade & $2(11.1)$ \\
\hline Subclinical (\%) & $2(11.1)$ \\
\hline I (\%) & $7(38.9)$ \\
\hline II (\%) & $7(38.9)$ \\
\hline III (\%) & $13.9 \pm 3.7$ \\
\hline Testes volume & $14.5(13-22.5)$ \\
\hline Last followup in months & \\
\hline
\end{tabular}

Mean \pm standard deviation, median (interquartile range 25-75), 


\begin{tabular}{|c|c|c|c|c|c|}
\hline Semen analysis & $\begin{array}{c}\text { Baseline } \\
n=18\end{array}$ & $\begin{array}{c}\sim 3 \text { months } \\
n=18\end{array}$ & $\begin{array}{c}\geq 12 \text { months } \\
n=18\end{array}$ & $\begin{array}{c}\text { Overall, } \\
\text { p }\end{array}$ & $\begin{array}{l}\text { Baseline vs. } \\
\geq 12, p\end{array}$ \\
\hline Volume & $2.6(2-3.4)$ & $2.5(1.9-4.4)$ & $3(1.2-4.2)$ & 0.988 & 0.791 \\
\hline TMSC & $6.4(1.1-24.5)$ & $11.1(2.4-38.4)$ & $12.5(1.6-31.5)$ & 0.849 & 0.650 \\
\hline Concentration & $10.7(3.5-21.3)$ & $14.5(4-22.6)$ & $16(1.4-20)$ & 0.728 & 0.563 \\
\hline Total motility & $36.7(10-52.5)$ & $38.5(12.3-60)$ & $49.5(31-62)$ & 0.395 & 0.143 \\
\hline
\end{tabular}

Median (interquartile range 25-75). TMSC: total motile sperm count. 